\title{
Development of a Neutron Radiography Three-Dimensional Computed Tomography System for Void Fraction Measurement of Boiling Flow in Tight Lattice Rod Bundles*
}

\author{
Masatoshi KURETA $^{* *}$ \\ **Nuclear Science and Engineering Directorate, Japan Atomic Energy Agency, \\ Tokai-mura, Naka-gun, Ibaraki-ken, 319-1195, Japan \\ E-mail: kureta.masatoshi@jaea.go.jp
}

\begin{abstract}
A neutron radiography three-dimensional computed tomography (NR3DCT) system was developed to visualize the void fraction distribution of boiling flow in tight lattice heated-rod bundles. This paper chiefly reports on the data processing and the error estimation method of NR3DCT. Practical $\gamma$-ray noise reduction and image correction techniques were studied to improve the reliability of the experimental data. Using the system and a directly heated 14-rod bundle test section, the behavior of boiling flow in a tight lattice rod bundle was clearly visualized. The effect of each data processing step on the result was also discussed. By this development, the three-dimensional vapor distribution of boiling flow in a heated bundle is made clear, and void fraction databases can be provided for verification of a thermal-hydraulic simulation code.
\end{abstract}

Key words: Void Fraction, Neutron Radiography, CT, Measurement, Boiling Flow, Visualization, Rod Bundle, Tomography

\section{Introduction}

The Japan Atomic Energy Agency (JAEA) has designed an innovative boiling water reactor named Innovative Water Reactor for Flexible Fuel Cycle (FLWR) ${ }^{(1)}$ in cooperation with reactor makers. For the reduction of the water fraction in the FLWR core, a tight-lattice fuel rod arrangement was adopted, and the reactor is operated with a high exit void fraction. Three-dimensional (3D) void fraction data were needed to verify a thermal-hydraulic simulation code ${ }^{(2)(3)}$. For this purpose, a Neutron Radiography Three-Dimensional Computed Tomography (NR3DCT) system was developed, and the 3D void fraction distribution in a tight lattice rod bundle was measured using the system.

Neutron radiography (NR) is a nondestructive testing technique, and has attracted the attention of several researchers and engineers ${ }^{(4)}$. Neutron tomography was developed based on NR, with a Computed Tomography (CT) technique. Neutron tomography has several advantages compared with the other void fraction measurement techniques, e.g. high sensitivity to water flowing in the metallic channel. Reviews of the research and development of neutron tomography include the following. Vontobel et al. ${ }^{(5)}$ studied geological material at the Paul Scherrer Institute in Switzerland. An energy-selective tomography was developed by Kardjilov et al. ${ }^{(6)}$ using the reactor FRM-I in Germany. A neutron phase-contrast tomography is under development by Dubus et al. ${ }^{(7)}$ at the Institute Laue-Langevin in France. Dierick summarized the NR and the neutron tomography well 
in his doctoral thesis ${ }^{(8)}$, in which many visualization results achieved at several neutron radiography facilities were reported. Takenaka et al. studied the behavior of the adiabatic air-water two-phase flow in a $4 \times 4$ rod-bundle and discussed the spacer effect in the research reactors JRR-3 ${ }^{(9)}$ and YAYOI ${ }^{(10)}$. Yamada et al. ${ }^{(1)}$ applied neutron tomography to the science of agriculture, specifically distribution of water in Japan cedar wood.

The Japanese government has promoted quantum beam technology since 2006. As the peaceful use of the neutron beam including neutron tomography is one quantum beam technology, JAEA is vigorously promoting the development of the neutron beam technology for many applications.

Since the void fraction measurement technique by neutron tomography had not been established, an original NR3DCT system was developed and void fraction tests were carried out.

The scopes of a series of our papers are as follows:

(1) NR3DCT system, data processing and error estimation method (present paper): The fundamental equations, equipment for NR3DCT, and the data processing involved are described systematically.

(2) Study on boiling flow in tight lattice rod bundles (2nd paper): Experimental study on boiling flow in the tight lattice 7- and 14-rod bundles will be described based on the $3 \mathrm{D}$ void fraction data with the NR3DCT system.

(3) Study on the behavior of air-water adiabatic two-phase flow and boiling flow in tight lattice rod bundles (3rd paper): Experimental study on the time-change of the instantaneous 2D void fraction distribution will be summarized based on high-frame-rate neutron radiography (HFR-NR) data.

\section{Fundamental Equations of the Void Fraction Measurement by NR3DCT}

Brightness, $B([\mathrm{x}][\mathrm{y}])$, of a NR image is in proportion to the penetrating neutron flux, $\phi_{\mathrm{p}}$, as follows :

$$
\begin{aligned}
& B=C \cdot \phi_{p}+B_{0} \\
& \phi_{p}=\phi_{t h} \cdot \exp \left(-\Sigma_{1} \delta_{1}-\Sigma_{2} \delta_{2} \cdots-\Sigma_{n} \delta_{n}\right) \\
& B_{0}=B_{s}+B_{d} \\
& B_{S}=C \cdot\left(\phi_{s m}+\phi_{s s}\right)
\end{aligned}
$$

where $C, B_{0}, \phi_{\mathrm{th}}, \Sigma, \delta, B_{\mathrm{s}}, B_{\mathrm{d}}, \phi_{\mathrm{sm}}, \phi_{\mathrm{ss}}$ denotes a constant which indicates the sensitivity of the imaging system, the offset term, the flux of the incident neutron beam, the total macroscopic cross-section of neutrons, the object thickness, the quantity of brightness increase caused by the scattered neutron, the flux of neutrons scattered in the object and the flux of neutrons scattered in the surrounding space such as the bio-shields, respectively. Neutron penetration ratio, $R[\mathrm{x}][\mathrm{y}]$, defined in Eq.(2), can be calculated by calibration with the no-object image, the so-called 'shading image', $B_{\mathrm{n}}[\mathrm{x}][\mathrm{y}]$, and the no-neutron-beam image, the so-called 'dark current image', $B_{\mathrm{d}}[\mathrm{x}][\mathrm{y}]$.

$$
R[x][y]=\frac{\phi_{p}[x][y]}{\phi_{t h}[x][y]}=\frac{B[x][y]-B_{d}[x][y]}{B_{n}[x][y]-B_{d}[x][y]}
$$

A CT value is calculated from a $(\Sigma \delta)$ value by the reconstruction method. Here, "reconstruction method" refers to creation of a cross-sectional image. The $(\Sigma \delta)$ value is calculated from Eqs. (1) and (2). The CT value corresponds to the total macroscopic cross-section, $\Sigma$, on the cross-sectional plane of the object. The $3 \mathrm{D}$ distribution of the CT value, $C T[\mathrm{x}][\mathrm{y}][\mathrm{z}]$ is obtained by piling up the cross-sectional planes. The $3 \mathrm{D}$ distribution of the void fraction, the so-called 'local void fraction', $\alpha[\mathrm{x}][\mathrm{y}][\mathrm{z}]$, is determined by Eq.(3) 
when the $\Sigma$ of gas-phase is negligibly small $\left(\Sigma_{\mathrm{g}} / \Sigma_{\mathrm{f}}=0.0006\right.$ under the present test conditions).

$$
\alpha[x][y][z]=1-\left(\frac{C T_{m}[x][y][z]}{C T_{f}[x][y][z]}\right)
$$

where $C T_{\mathrm{m}}$ and $C T_{\mathrm{f}}$ denote the $\mathrm{CT}$ values calculated from the boiling flow data and the fluid-filled test area data, respectively. The simple fundamental equations are as described in this chapter. However, many kinds of data collection are needed to obtain reliable data.

\section{Neutron Imaging System}

The NR imaging system was installed at a thermal NR facility with a thermal neutron beam flux, $\phi_{\mathrm{th}}$, of $1.5 \times 10^{8} \mathrm{n} /\left(\mathrm{cm}^{2} \mathrm{~s}\right){ }^{(12)}$ in the JRR-3. The NR3DCT imaging system was constructed with a neutron-to-fluorescence converter made from ${ }^{6} \mathrm{LiF} / \mathrm{ZnS}: \mathrm{Ag}$, a high sensitivity CCD digital camera (Hamamatsu Photonics, Model C4880), a stepping motor, and a control computer. In this section, the hardware and the software of the NR3DCT is described.

\subsection{Neutron Tomography System}

Figure 1 shows the schematic diagram of the NR3DCT system. A NR image was automatically recorded on the control computer. An image was 1008(W) x 1023(H) pixels of 14 bit (16384 steps gray scale) non-compressed binary data. The maximum brightness of the image was set to be the highest non-saturated value to keep higher dynamic range, by adjusting the recording time in advance. In order to get the 3D data, in present NR3DCT experiments, an object (test section, samples etc.) was revolved up to 180 degrees, and the images were recorded at each projection angle. The stepping motor was installed at the upper part of the test section for boiling experiments. Control accuracy of the motor was 0.01 degree. The vertical position of the test section was adjusted to the target position using a mechanical fine control system. The projection angle and the recording of the images were automatically controlled by the control computer. The irradiation time for one frame was ranged from 0.9 to $3.5 \mathrm{~s}$. The object stood still when the image was recorded. Figure 2 shows the experimental apparatus setup in the neutron radiography room. The neutron beam penetrated the test section, and the projection image on an NR converter was recorded by the camera after reflection by the double mirrors.

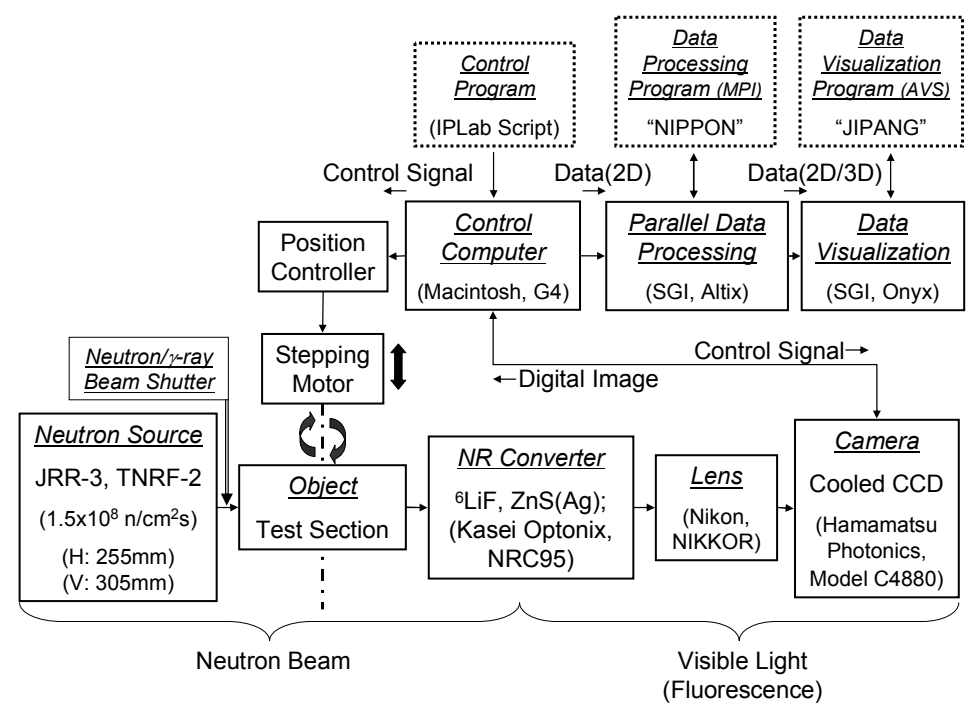

Fig. 1 Schematic diagram of the NR3DCT system 


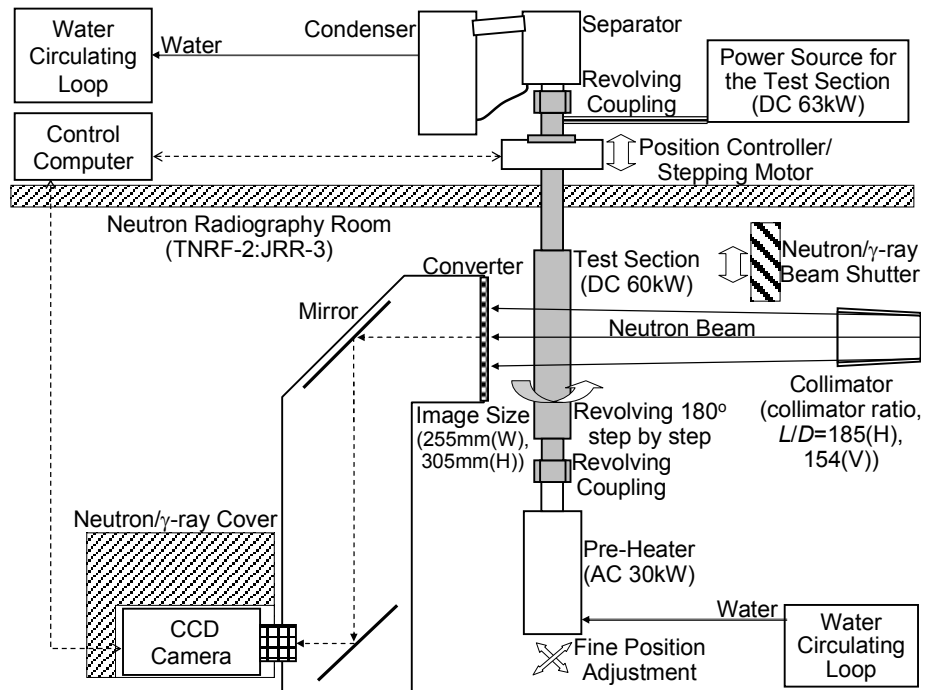

Fig. 2 Experimental setup in the neutron radiography room

Figure 3 shows the schematic view of a 14-rod bundle test section. This test section simulated the FLWR core. The heater power was made sufficient to visualize the void fraction from the vapor generation to the high void fraction region at the same time. The recording time for one condition depended on the number of projections done etc. In case of 180 steps $/ 180$ degrees, the 180 projection images were recorded in about forty minutes. The recorded images were transferred to a parallel processing computer for the quantitative processing as shown in Fig. 1.

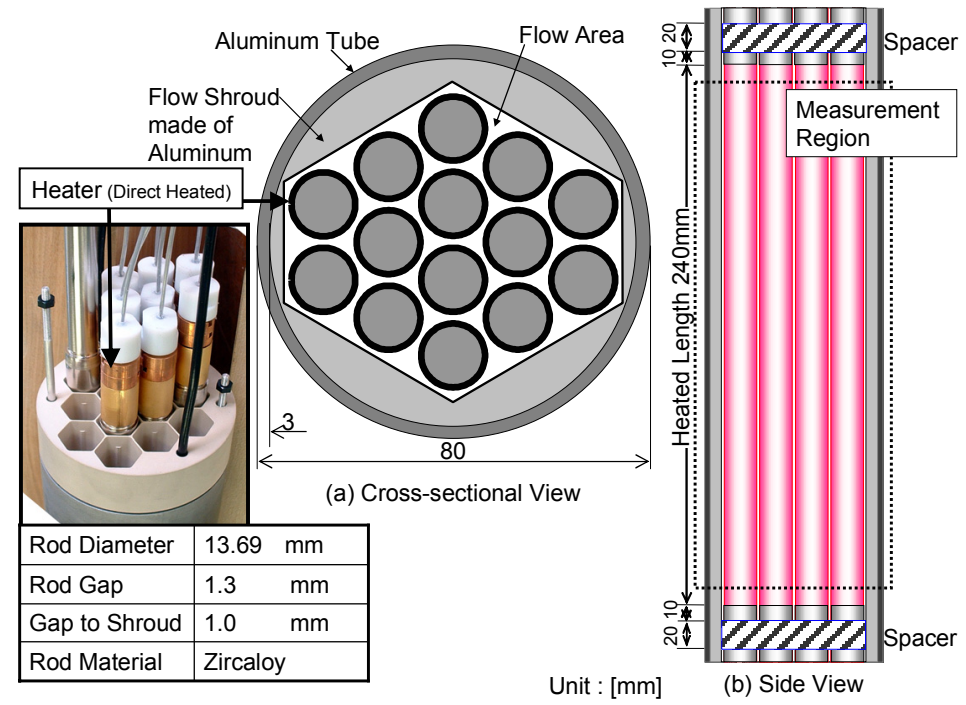

Fig. 3 Schematic view of a 14-rod bundle test section

\subsection{Fundamental Setup for the Neutron Tomography}

\subsubsection{Setup position of the test section}

In order to record higher quality NR images, the following settings were made:

(1) The test section and the converter were aligned at right angles to each other, facing the neutron beam. That is, the test section was fixed vertically at the beam center with high accuracy using the vertical position controller. (2) The distance between the test section and the converter, $L_{\mathrm{ts}}$, was an important parameter for obtaining a sharp image. Sharpness of the image is largely dependent on controlling the divergence of the incident neutron beam ${ }^{(13)}$ and the scattering of the neutron beam in the test section, and on the focus adjustment of the imaging system. The focus adjustment of the imaging system was best 
regulated using visible light. If the total macroscopic scattering cross-section of the test section is negligibly small, the clearest image would be obtained at $L_{\mathrm{ts}}=0$, that is, when the test section is in contact with the converter. However, when the test section included high scattering cross-section material such as water, a better image was obtained at $L_{\mathrm{ts}}=$ (from 0.5 to 3 ) $x$ (diameter of the test section) in case of the JRR-3 NR facility. Hibiki et al. ${ }^{(14)}$ studied the influence of scattered neutrons on the NR image. The present pre-test data and Hibiki's results were similar. Therefore, the distance for the best sharpness was found by the pre-test.

\subsubsection{Marker for the corrections}

Two types of markers, indicating inclination and image center, were used to correct the brightness, inclination and rotating axis. Figure 4 shows a sample (digital still camera) NR image with the markers ' $\mathrm{A}$ ' and ' $\mathrm{B}$ '. In the image, a rectangular plate with four holes (plate-type marker; ' $A$ ') and circular markers (ribbon-type marker; 'B') are indicated. The markers ' $\mathrm{A}$ ' and ' $\mathrm{B}$ ' were made of cadmium and $\mathrm{BN} /$ polyethylene, respectively. The maker 'A' was used for the brightness correction and to calculate the spatial resolution, $S R$ $[\mathrm{mm} / \mathrm{pixel}]$. Specifically, the center point of the hole was defined as the standard point for the brightness correction, and the $S R$ was calculated from the distance between the centers of two holes. On the other hand, the markers 'B' surrounded the test section as shown in Fig. 4. Since the line through the centers of the two ribbon circles was kept to be the rotating axis, the correction angle for the inclination correction and the clipping region for the centering correction were calculated semi-automatically by using these marker techniques.

\subsection{Data Processing System}

All NR3DCT images were processed using a data processing program (NIPPON), and were visualized by a visualization program (JIPANG). The NIPPON was designed for the high-performance parallel processing computer system of JAEA. JIPANG was developed for the $2 \mathrm{D}$ and $3 \mathrm{D}$ visualization of the data. These programs were developed not only for the NR3DCT but also for the HFR-NR.

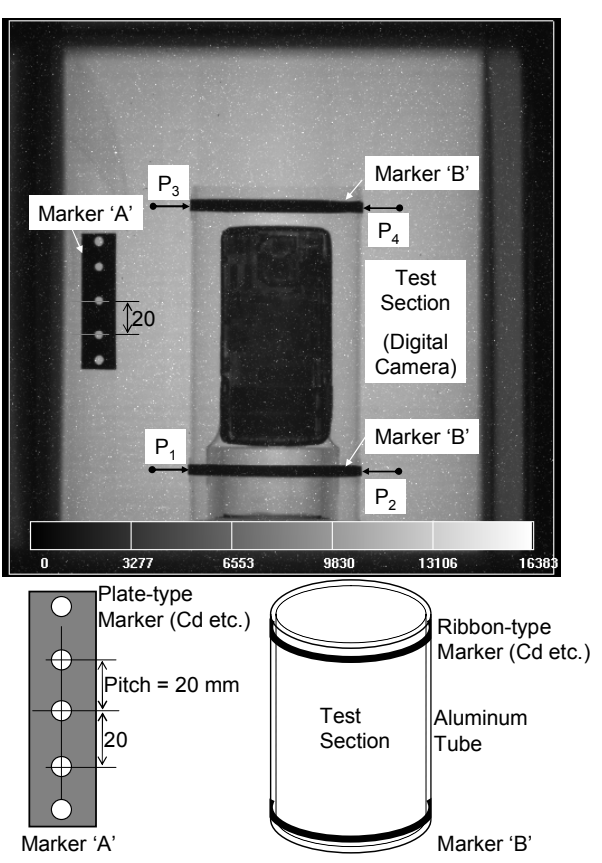

Fig. 4 Sample image recorded with the markers

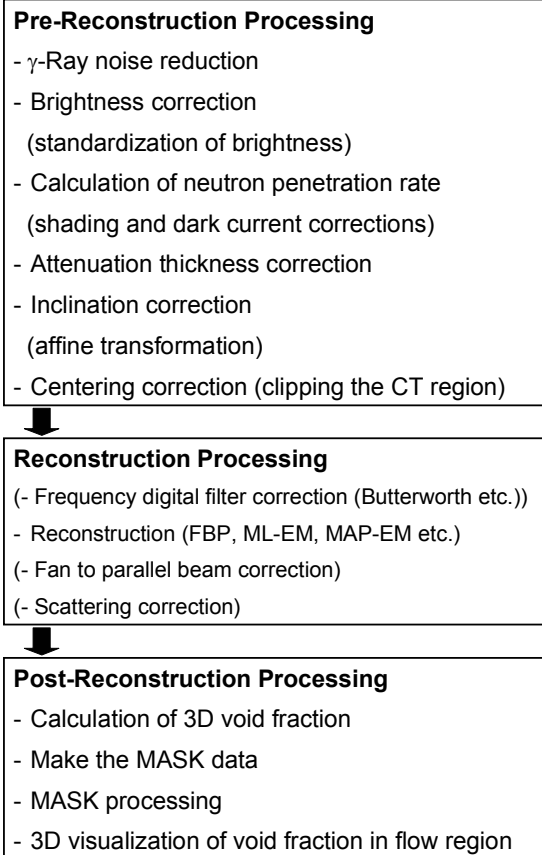

Fig. 5 Data processing procedure of the NR3DCT 


\section{Data Processing Methods}

Figure 5 is a flow diagram of the data processing programmed in NIPPON. The data processing methods from an original NR image to the $3 \mathrm{D}$ void fraction distribution by the NR3DCT are discussed below in sequence.

\subsection{Pre-Reconstruction Processing}

\subsubsection{Noise reduction}

On an original NR image recorded by a very high sensitivity cooled CCD imaging system, white/gray noise was uniformly added on the image as shown in Figs. 4 and 6(a). Since the noise was caused by $\gamma$-rays, it should be reduced in the first stage of the data processing.

(A) Characteristics of $\gamma$-ray noise: Figure 7 shows the typical noise pattern. In a detailed analysis of the noise, the following characteristics were found: (1) the noise was independent of the neutron penetration rate, and (2) the noise was not so-called 'white noise' but rather clusters were formed. It appears that a $\gamma$-ray beam charged not only one $\mathrm{CCD}$ element but also the surroundings at the same time.

(B) Noise reduction method: At first, an 8-neighborhood smoothing filter that replaced the target value with mean value, a median filter and a digital frequency filter (Butterworth filter) were examined for their noise reduction. It was found that the noise was not reduced perfectly because of the clustering and the high brightness points. Also, the sharpness of the image was decreased. In order to reduce the noise more effectively, various noise reduction methods were tested using real images recorded under different conditions. In consequence, it was found that the following data processing achieved effective noise reduction:

(1) Create a $5 \times 5$ matrix around a target point $P[x][y]$.

(2) Calculate standard deviation, $s$, in the area of 8 neighborhoods by the following equations;

$m=\frac{1}{9} \sum_{i=6}^{18} c[i], s^{2}=\frac{1}{9} \sum_{i=6}^{18}(c[i]-m)^{2}, s=\sqrt{s^{2}}$

where $i, c[i]$ denote one of the cells around the target point $P[x][y]$ numbered from 0 to 24 and the cell value at $i$, respectively. The value at the $P[x][y]$ is $c[12]$ in this $5 \times 5$ matrix.

(3) Decide whether there is noise around the $P[\mathrm{x}][\mathrm{y}]$. If $s$ is smaller than the threshold, then the program decides that there is no noise and the cell remains at its original value, $c[12]$. If not, then the program decides that the $P[x][y]$ is in a noise area and proceeds to the next process (4). Here, the threshold is set to 64 based on a parameter survey.

(4) Sort the $5 \times 5$ matrix and replace the original pixel value with the mean value of the median 5 values expressed as;

$N e w[x][y]=\frac{1}{5} \sum_{i=10}^{14} c_{\text {sort }}[i]$

where $N e w[\mathrm{x}][\mathrm{y}], i$, and $c_{\text {sort }}[i]$ denote the replacement pixel value, a number from 0 to 24 , and the value of the $i$ th pixel where the pixels have been numbered successively from least to greatest value, respectively.

(C) Result of the new noise reduction method: Comparison of the brightness distribution of an original image and the noise reduced image is shown in Fig. 6. In the red rectangular boxes, macrographs of the images are given. Figure 6(b) gives the noise reduced image of the right-half-area of Fig. 6(a). Figures 6(a') and (b') show the surf plots of the original and the noise reduced data, respectively. It is evident from Fig. 6 that the $\gamma$-ray noise was reduced well and the sharpness was maintained effectively. 

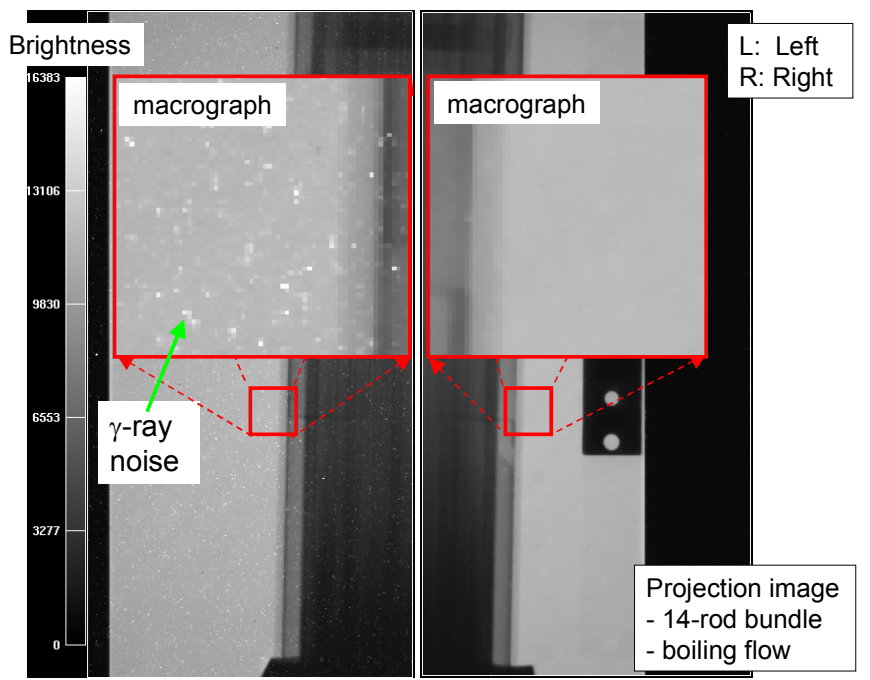

(a) Original image (L)

(b) Noise reduced image ( $R$ )

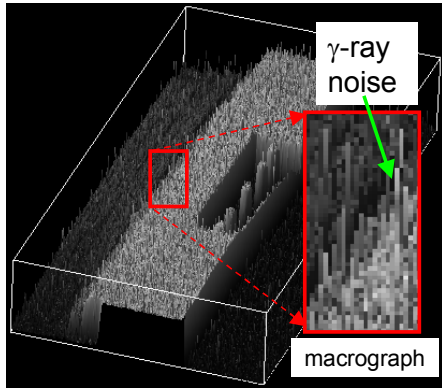

(a') Surf plot of the original image $(R)$

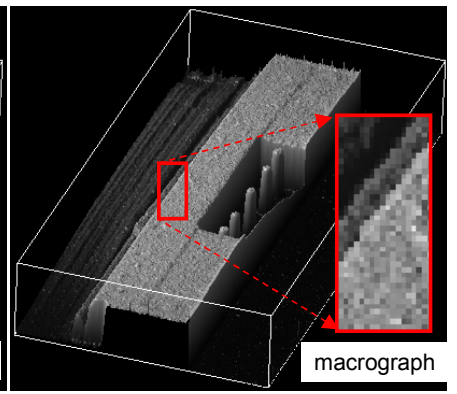

(b') Surf plot of the noise reduced image $(R)$

Fig. 6 Original neutron radiography image and noise reduced image

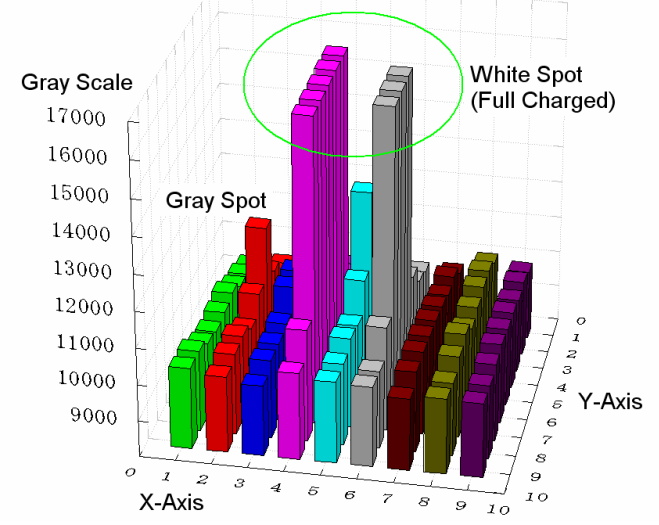

Fig. 7 Typical noise pattern (cluster-type)

\subsubsection{Brightness correction}

The steady neutron beam generated in the research reactor was used. However, the neutron flux on the converter was slightly changed because of change in the reactor core conditions. Also, the output intensity of an imaging system normally drifts. Therefore, brightness correction was needed for the quantitative evaluation. Since the change of the spatial distribution was negligibly small, following brightness correction was conducted in the NIPPON:

(1) Decide the standard brightness, $B_{\mathrm{STD}}$, at a point removed from the object, $\left(x_{\mathrm{STD}}, y_{\mathrm{STD}}\right)$. In this test, we set the center point of the hole of the Marker ' $\mathrm{A}$ ' as $\left(x_{\mathrm{STD}}, y_{\mathrm{STD}}\right)$.

(2) Calculate a brightness correction factor, $k_{\mathrm{b}}$, for all images as follows;

$$
k_{b}=B / B_{S T D}
$$


where $B$ is the brightness at $\left(x_{\mathrm{STD}}, y_{\mathrm{STD}}\right)$.

(3) Correct all pixel values using $k_{\mathrm{b}}$;

$$
B_{N E W}[x][y]=\frac{1}{k_{b}} B_{O R G}[x][y]
$$

Figure 8 shows a typical angular distribution of $1 / k_{\mathrm{b}}$ in Eq. (7) while the test section was rotating (about 40 minutes). This change was probably caused by the reactor core condition, the imaging system, the sampling time of the $B_{\mathrm{STD}}$ and the converter condition.

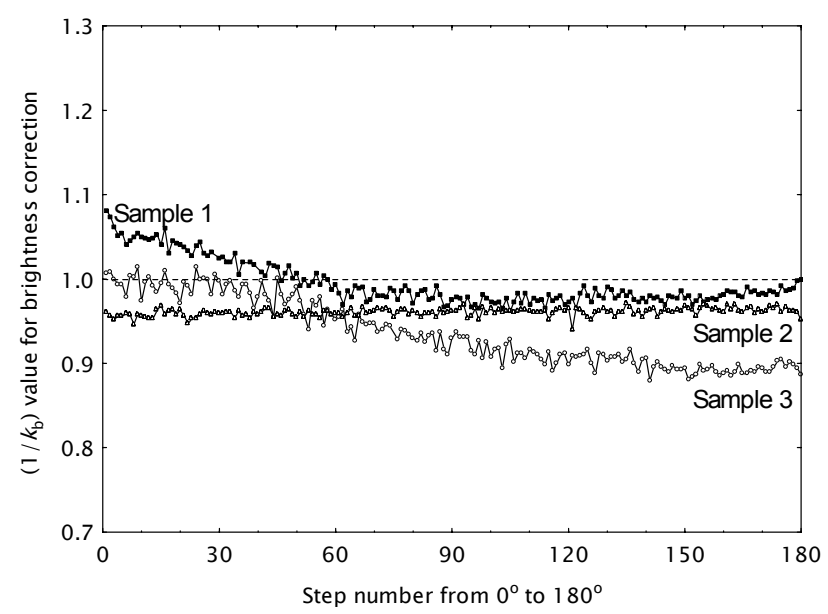

Fig. 8 Typical angular distribution of the brightness at the out of object point

\subsubsection{Neutron penetration rate}

The neutron penetration rate, $R$, was calculated by Eq. (2). This processing included the so-called 'shading correction' and the 'dark current correction'.

\subsubsection{Attenuation thickness correction and the $(\Sigma \delta)$ value}

Since the $R$ is equal to ' $\exp (-\Sigma \delta)$ ', the distribution of the $(\Sigma \delta)$ value can be calculated by Eq. (8);

$$
\left(\sum \delta\right)[x][y]=-\ln (R[x][y])
$$

Yokoi et al. ${ }^{(15)}$ pointed out that an attenuation thickness correction of the $\Sigma$ was needed when the $\Sigma$ depended heavily on the attenuation thickness, $\delta$, and the beam spectrum. Therefore, $R$ also is a function of the $\delta$. Since the target object was water in present project, the attenuation thickness correction by using the reverse function method proposed by Oda ${ }^{(15)}$ was applied. In this study, nonlinear fitting function of the $R$ was decided as follows;

$$
R(\delta)=\exp \left[-\Sigma_{0}\left(1-K_{m} \delta\right) \delta\right]
$$

where $\Sigma_{0}$ and $K_{\mathrm{m}}$ denote the extrapolated macroscopic cross-section at $\delta=0$ and the fitting parameter, respectively. Equation (9) can be rewritten for reconstruction processing the same way as Eq. (8);

$$
\left(\Sigma_{0} \delta\right)[x][y]=\frac{1-\sqrt{1+4\left(K_{m} / \Sigma_{0}\right) \ln (R[x][y])}}{2\left(K_{m} / \Sigma_{0}\right)}
$$

In this study, this $\left(K_{\mathrm{m}} / \Sigma_{0}\right)$ value was decided to be 0.109 by nonlinear fitting using polystyrene plates with the thickness ranging from 1.0 to $16.7 \mathrm{~mm}$. Since the neutron scattering characteristic of water is similar to that of polystyrene $\left(\Sigma_{\text {polystyrene }}=2.12 \mathrm{~cm}^{-1}\right.$, $\left.\Sigma_{\text {water }}=3.45 \mathrm{~cm}^{-1}\right)$, the attenuation correction factor $\left(K_{\mathrm{m}} / \Sigma_{0}\right)$ for water was thought to be similar to that of polystyrene. Reconstruction processing was needed to calculate the $3 \mathrm{D}$ distribution of the $2[\mathrm{x}][\mathrm{y}][\mathrm{z}]$. Here, the so-called ' $\mathrm{CT}$ value' calculated by the reconstruction processing corresponds to the $\Sigma$. 


\subsubsection{Inclination and centering corrections, and cut out of the $\mathrm{CT}$ region}

In order to calculate the accurate $\mathrm{CT}$ image by the reconstruction processing, the rotating axis line had to be on the midpoint of the X-direction-width of the pre-reconstruction image. The projected image of the test section was inclined a little because of the limitation of the manual mirror adjustment. Figure 9 shows the flow of the data correction processing process. The inclination was corrected by the following affine transformation;

$$
\begin{aligned}
& X=\left(x-x_{0}\right) \cos \theta+\left(y-y_{0}\right) \sin \theta+x_{0} \\
& Y=-\left(x-x_{0}\right) \sin \theta+\left(y-y_{0}\right) \cos \theta+y_{0}
\end{aligned}
$$

where $(X, Y),\left(x_{0}, y_{0}\right)$ and $\theta$ denote the transformed point, the rotating center and the rotating angle (counterclockwise), respectively. Here, the enlargement/reduction factor was set to 1. After the determination of the test section area on the inclination corrected image, a centering correction and a cut out processing of the $\mathrm{CT}$ region were conducted as illustrated in Fig. 9, before the reconstruction processing.

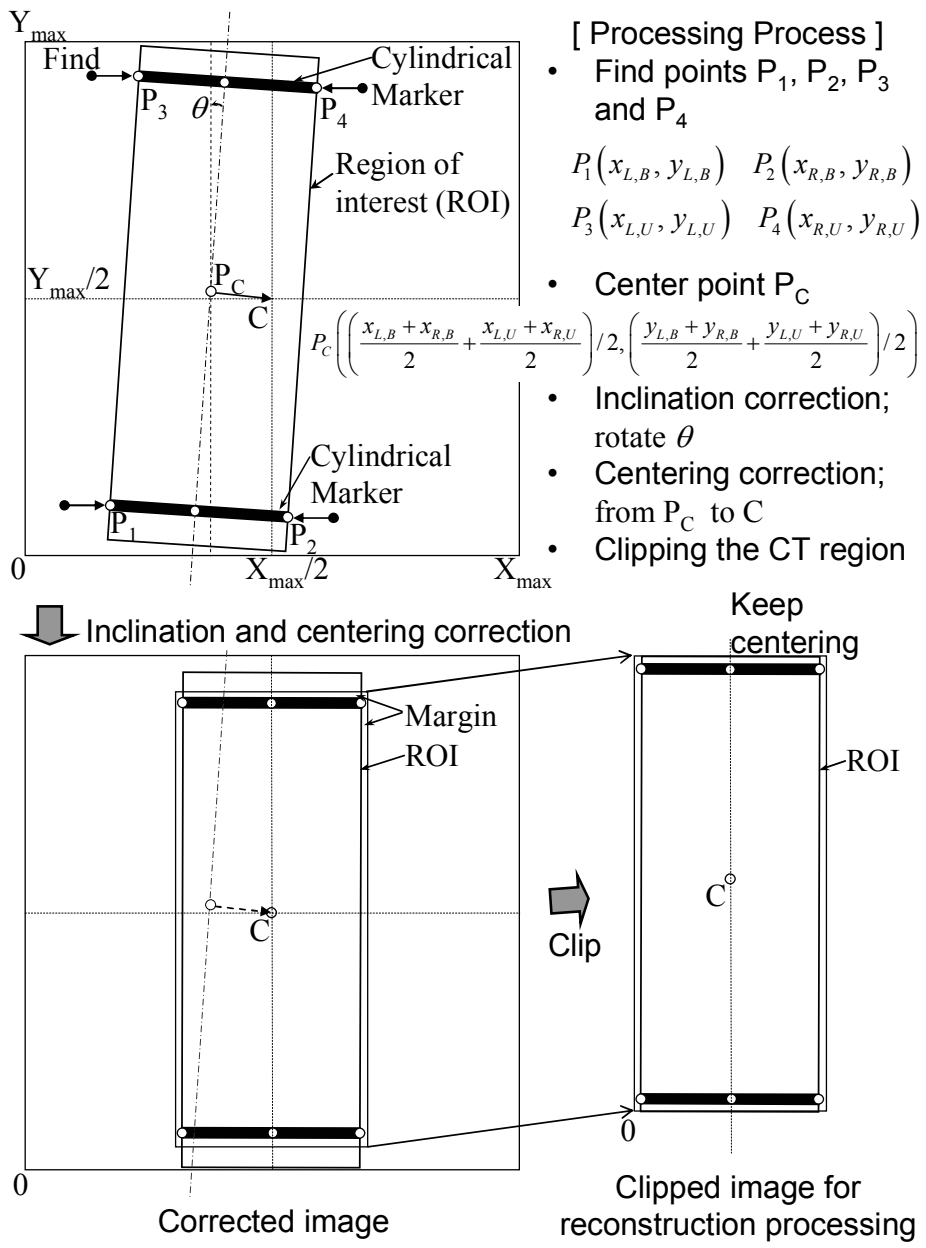

Fig. 9 Inclination, centering and ROI clipping methods

Figure 10 demonstrates the effect of the centering correction on the CT image. When the center line of the rotating does not lie on the midpoint of the X-direction-width of the pre-reconstruction image as displayed in Fig. 10(A) and (B), the upper and lower radius on the calculated CT image were different from each other. After the appropriate centering correction and cut out of the CT region, a suitable CT image could be calculated as shown in Fig. 10(C). 

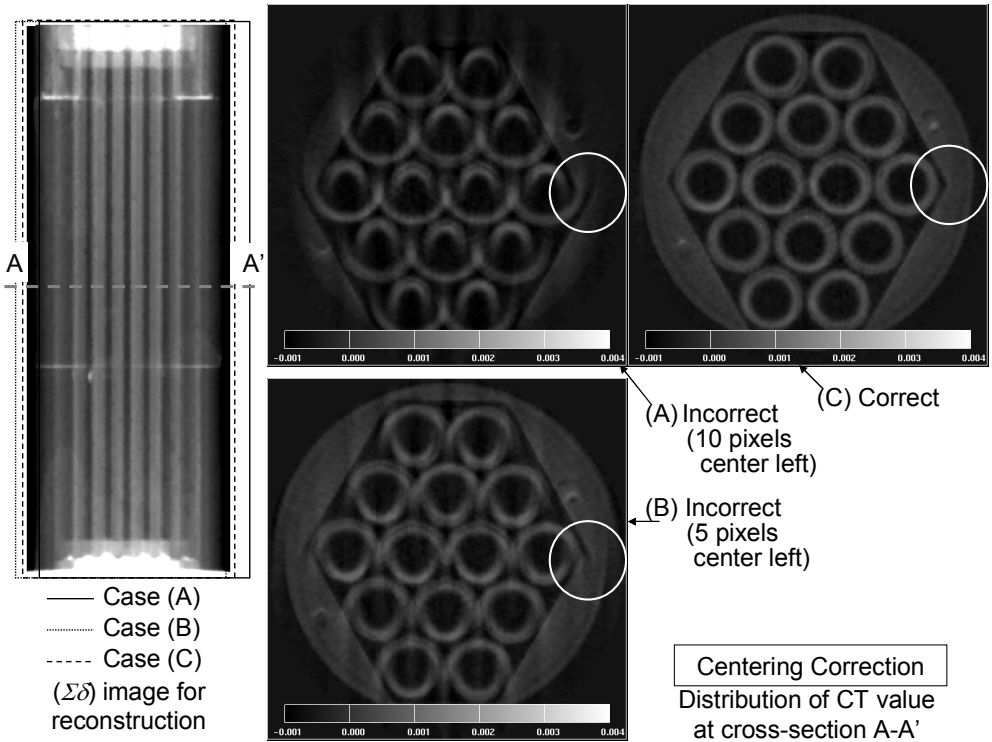

(A) Incorrect
(10 pixels
center

center left)

(B) Incorrect

(5 pixels

center left)

Fig. 10 Effect of centering correction on CT image

\section{Centering Correction Distribution of CT value at cross-section A-A'}
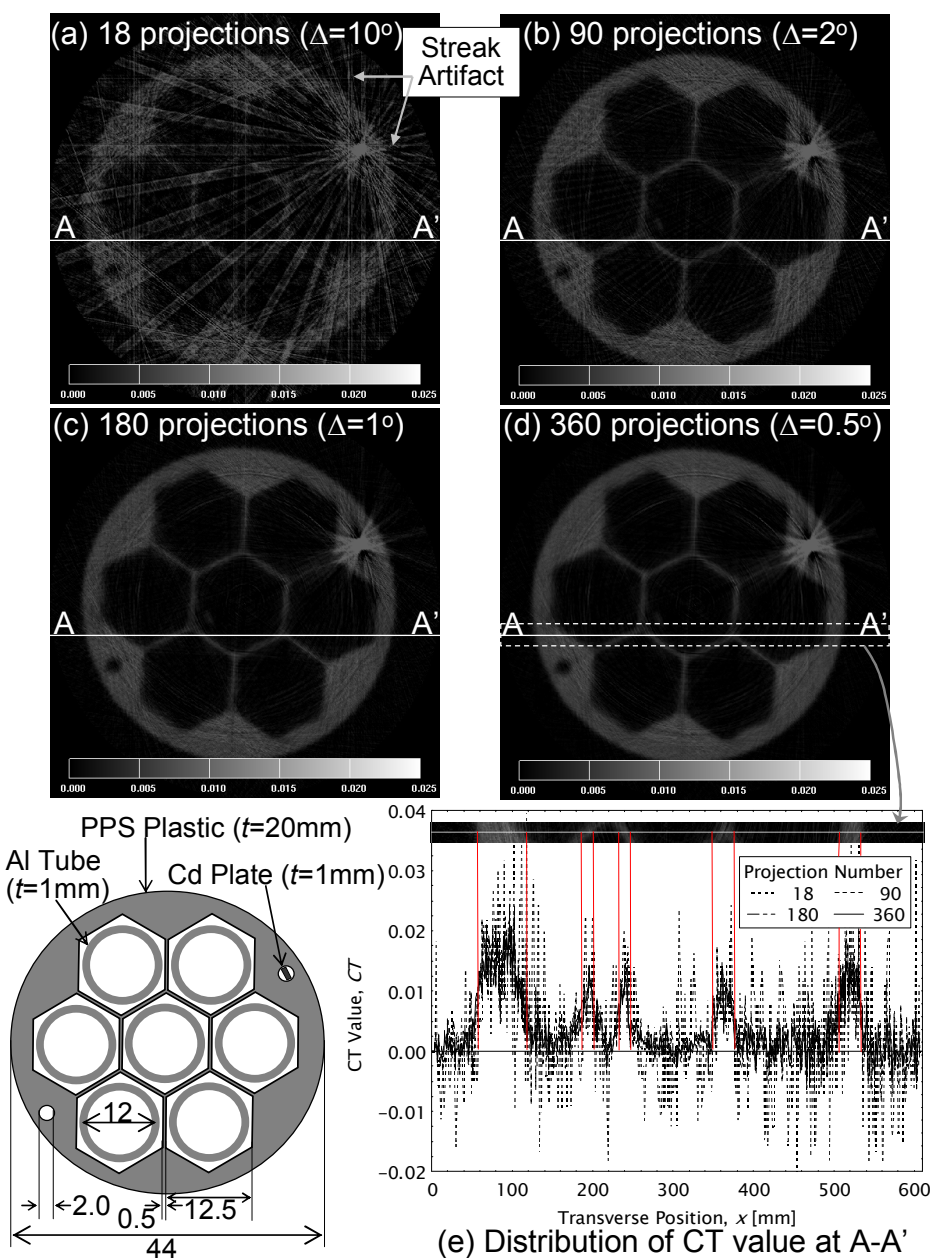

(e) Distribution of CT value at A-A'

Fig. 11 Relation between reconstruction result and projection number

\subsection{CT Processing}

Reconstruction methods can be classified roughly into two categories: (1) an analytical method, and (2) a probabilistic method. In this paper, a 'Convolution Filtered Back-Projection 'FBP' method is discussed. The FBP method is a representative analytical method. Because of the short calculation time and good sharpness of the reconstructed image, the FBP method is widely used in the X-ray CT system etc. 
First, the Hamming filter, Ram \& Lak filter and Shepp \& Logan filter ${ }^{(17)}$ were examined to select the filter with the best function for the FBP method. The Shepp \& Logan filter was selected because this filter maintained good spatial resolution and reduced the reconstruction noise most effectively. Since the quality of the reconstruction image was related to the projection number, the relation between the quality of the projected image and the projection number $\left(18,90,180,360\right.$ steps $\left./ 180^{\circ}\right)$ was studied as shown in Fig. 11. The quality of the reconstructed image became higher when the projection number increased. From this study, we selected $1^{\circ}$ as the projection step angle in our standard setting. When the projection step was smaller than $1^{\circ}$, since artifact noise distributed uniformly over the reconstructed image, the quality increase was small. The CT value must be a positive number because the value corresponds to the $\Sigma$. But at some calculated points, a negative number was calculated. This problem was caused by the characteristics of the filter function. In order to calculate the void fraction with more high accuracy, this negative number was replaced with 0 . Otherwise, a strange void fraction would be calculated at this abnormal point.

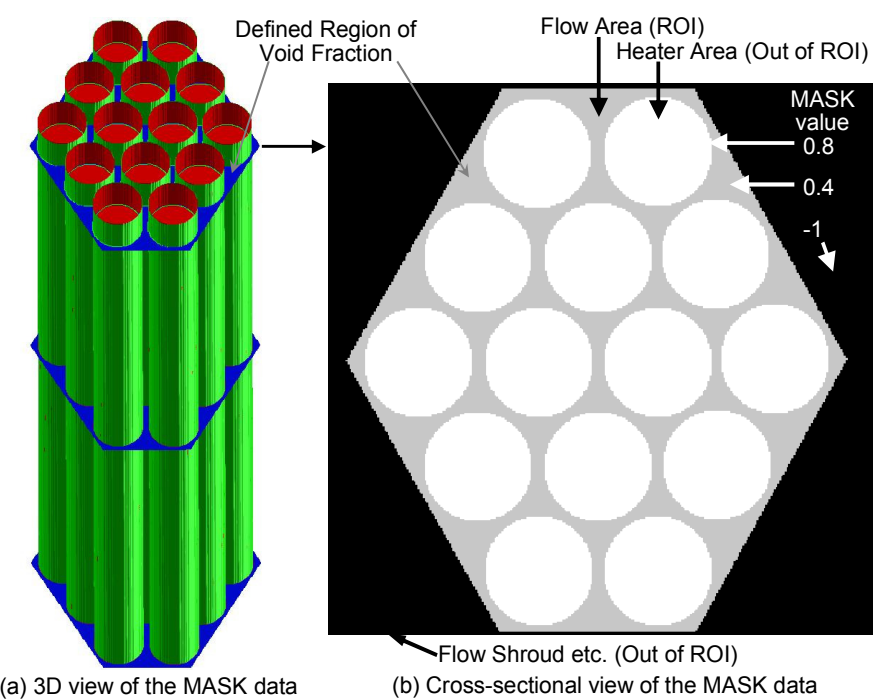

Fig. 12 MASK data for the 14-rod bundle test section

\subsection{Post-Reconstruction Processing}

The $3 \mathrm{D}$ void fraction distribution, $\alpha[x][y][z]$, in the flow area was calculated in the post-reconstruction processing using Eq. (3). Because the calculated value at areas other than the flow areas did not make sense, we replaced these meaningless values with a special value, -1 . Further, these points were set to be invisible in the data viewer JIPANG. The author named the replacement processing 'MASK processing', and the 3D data for the MASK processing was called 'MASK data'. Figure 12 shows the MASK data for a 14-rod bundle test section. The MASK data was calculated using the gas-filled test section data. The regions of the structures and the flow areas were distinguished by the CT value. Then we set these region identification numbers in the MASK data as indicated in Fig. 12. Figure 13 shows the typical 3D void fraction distribution in the flow area taken in the boiling flow experiment. In Fig. 13(a), the half-cut view is shown as a slice contour plot, and the vapor regions $(\alpha>0.9)$ were visualized as an isosurface plot. The cross-sectional distributions at elevations from the heater inlet $Z$ of 13, 123.5 and $229 \mathrm{~mm}$ were shown by surf plots in Fig. 13 b1-b3. The spatial resolution was $0.221 \mathrm{~mm} /$ voxel. The detailed vapor distribution of boiling flow in a heated bundle was elucidated, and new void fraction databases were provided for verification of advanced numerical analysis codes. Discussion of the HFR-NR data from the points of view of thermal-hydraulic science and engineering will be reported in following papers. 


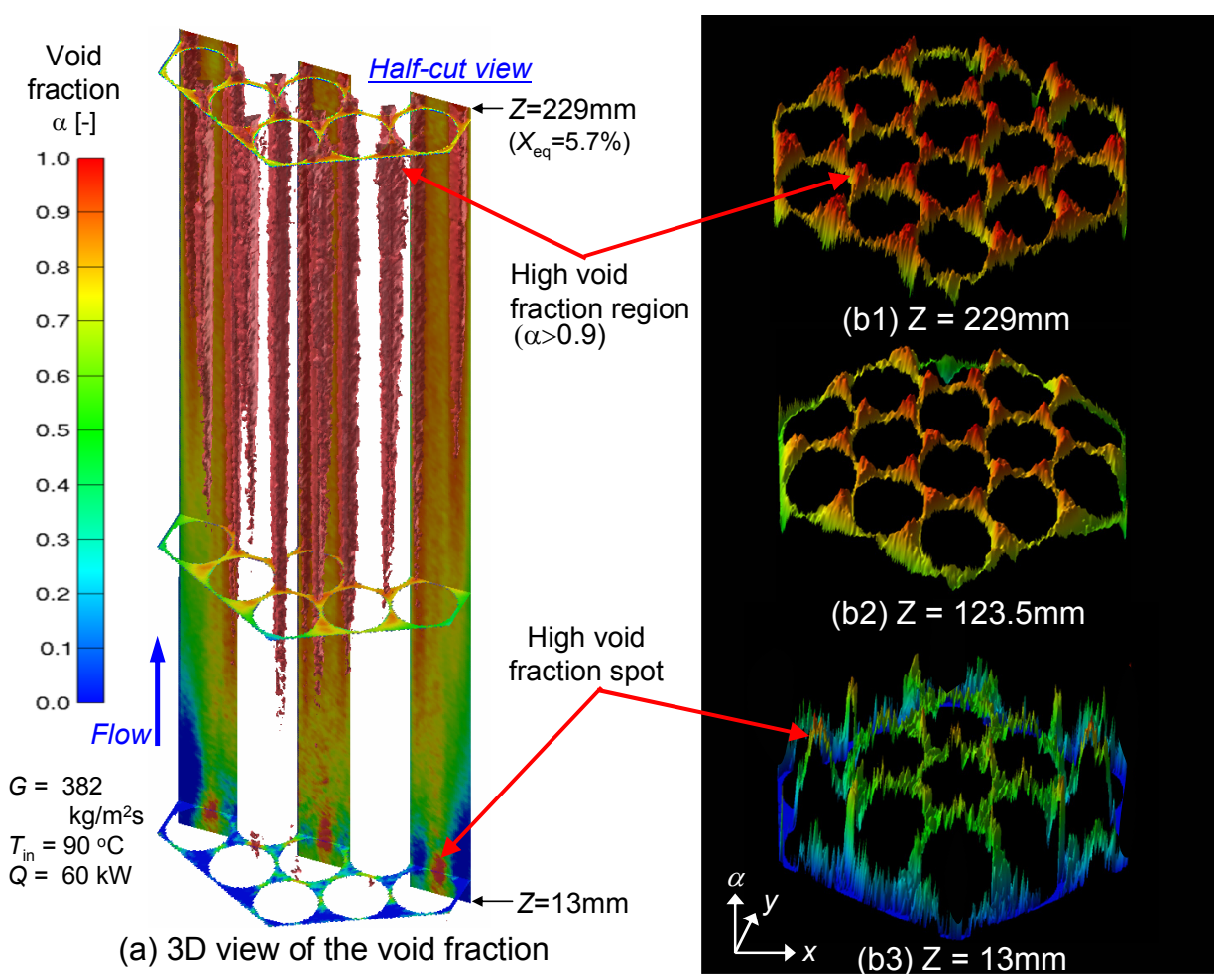

Fig. 13 Visualization of the void fraction distribution

\section{Estimation Method of Measurement Error}

An estimation of the measurement error of the void fraction calculated by the NR3DCT is needed, but had never been reported.

It was found from basic pre-tests that the measurement error increased remarkably when $\Sigma \delta$ was larger than about 3 because of the low neutron penetration rate, $R<5 \%$. In case of water, the error estimation was very difficult when the maximum penetration thickness was larger than approximately $1 \mathrm{~cm}$, because the scattering of the CT value significantly increased. This degradation of the data was mainly caused by strong artifact noise generated according to the structure of the test section.

In this study, a new error estimation method named 'high-speed revolving method' was developed for the low $(\Sigma \delta)$ region $(\Sigma \delta<3)$. Figure 14 is a schematic view of the method and evaluation results of this method. A key idea of this method was that the time-averaged void fraction could be set by the cut-out ratio of the high speed rotating test section. The test section was made of copper $\left(\Sigma_{\mathrm{Cu}}=0.924 \mathrm{~cm}^{-1}\right)$ with a diameter of $30 \mathrm{~mm}$ $(\Sigma \delta=2.8)$ in order to simulate water with the thickness of $8 \mathrm{~mm}$. The reason why the test material was made of copper was that its size and weight were then suitable for revolving the pieces without vibrating the test section. In this test, the pieces were revolved at rates ranging from $250 \mathrm{rpm}$ to $2,000 \mathrm{rpm}$. The designed void fractions were $0,0.25,0.5,0.75$, and 1. The revolving speed had no influence on the result in this range. The calculated value showed good agreement with the design value as shown in Fig. 14(b). The reliable range was about mean void fraction $\alpha \pm 0.05$, and was become smaller with increase in the void fraction.

It was confirmed from the error estimation that the void fraction in the homogeneous material could be measured within about $\alpha \pm 0.05$ under the condition $\Sigma \delta<3$. The error increased significantly around a strong neutron absorbing/scattering material such as water or under the condition of $\Sigma \delta>3$. 

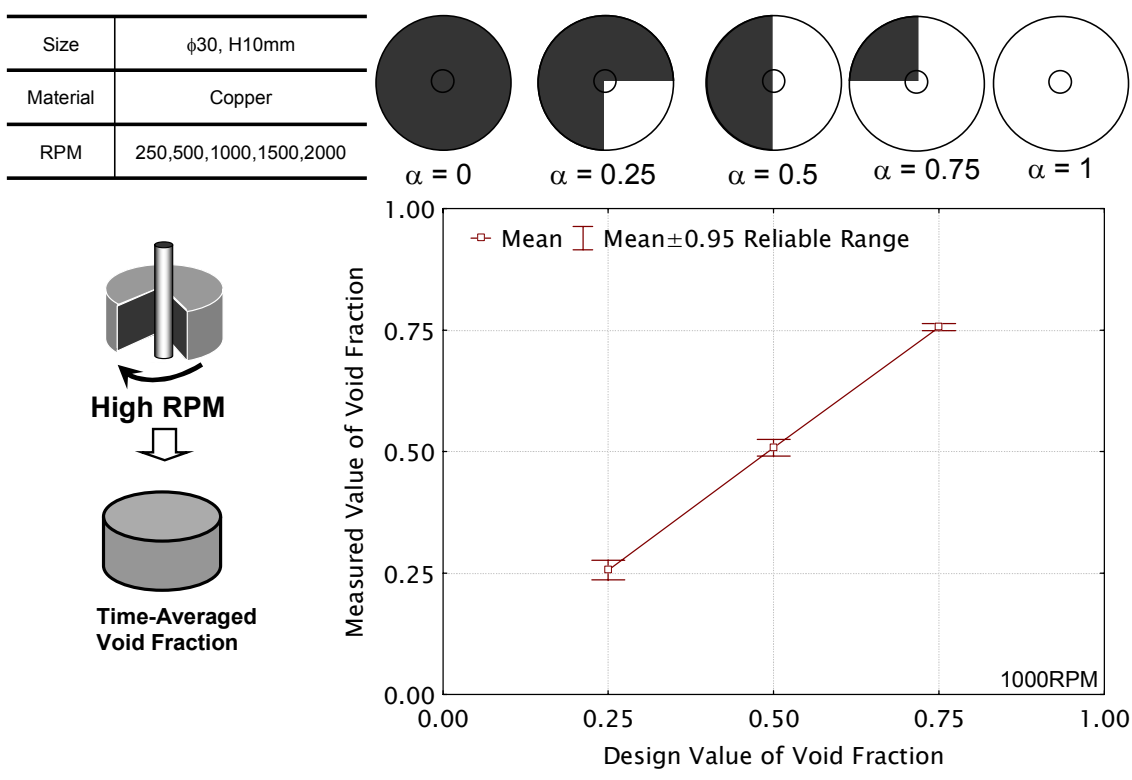

(a) Schematic view of high-speed revolving method

(b) Evaluation results of the NR3DCT by high-speed revolving method

Fig. 14 Evaluation of measurement error by high-speed revolving method

\section{Conclusions}

In order to measure and visualize the three-dimensional (3D) void fraction distribution of boiling flow in a tight lattice heated-rod bundle, a neutron radiography 3D computed tomography (NR3DCT) technique was developed as a new flow measurement technique. The system and the practical data processing methods were studied comprehensively. The reliability of the developed NR3DCT was discussed and was estimated by the new error estimation method named "high-speed revolving method" for the condition of $(\Sigma \delta)<3$. The 3D void fraction distribution of boiling flow in a tight-lattice 14-rod heated bundle could be measured and visualized with the spatial resolution of $0.221 \mathrm{~mm} / \mathrm{voxel}$ using the research reactor JRR-3 with the developed system. The vapor distribution in a heated bundle was elucidated by this study. Further, the 3D void fraction databases for verification of an advanced numerical analysis code can be provided.

\section{Acknowledgment}

The present study includes the results of "Development of Fuel Assembly for Very High Burn-up Water-cooled Breeding Reactor" commissioned through the Japan Atomic Energy Agency by the Ministry of Education, Culture, Sports, Science and Technology of Japan (MEXT). Much advice was given by H. Akimoto. Present experiments were carried out at JRR-3 of JAEA with the help of many able supporting staff members. This research was conducted using a supercomputer of JAEA.

\section{References}

(1) Uchikawa, S. et al., Conceptual design of Innovative Water Reactor for Flexible Fuel Cycle (FLWR) and its recycle characteristics, J. Nuclear Science and Technology, Vol. 44, No. 3 (2007), pp.277-284.

(2) Yoshida, H. et al., Verification of a detailed two-phase flow analysis code based on an advanced interface-tracking method (I) - Detail of numerical method and analysis of liquid film falling down on inclined flat plate -, Proc. of NUTHOS-6 (2004), Paper No. 
N6P266.

(3) Yoshida, H. et al., Development of predictable technology for thermal/hydraulic performance of reduced-moderation water reactors (3) - Current status of development of three-dimensional two-phase flow simulation method -, Proc. of ICAPP'04 (2004) Paper No. 4057.

(4) Selected papers of the WCNR-7 (2002), IEEE Trans. Nuclear Science, Vol. 52, No.1 (2005), pp.279-409.

(5) Vontobel, P., Lehman, E. and Carlson, W. D., Comparison of X-ray and neutron tomography investigations of geological materials, IEEE Trans. Nuclear Science, Vol. 52, No.1 (2005), pp.338-341.

(6) Kardjilov, N., Schillinger, B. and Steichele, E., Energy-selective neutron radiography and tomography, Applied Radiation and Isotope, Vol. 61 (2004), pp.455-460.

(7) Dubus, F. et al., First phase-contrast tomography with thermal neutrons, IEEE Trans. Nuclear Science, Vol. 52, No.1 (2005), pp.364-370.

(8) Dierick, M., Tomographic imaging techniques using cold and thermal neutron beams, Doctor thesis of Univ. Gent (2005).

(9) Takenaka, N. et al., Three-dimensional visualization of void fraction distribution in steady two-phase flow by thermal neutron radiography, Nuclear Engineering and Design, Vol. 184 (1998), pp.203-212.

(10) Takenaka, N. et al., Application of fast neutron radiography to three-dimensional visualization of steady two-phase flow in a rod bundle, Nuclear Instrument and Methods in Physics Research A, Vol. 424 (1997), pp.73-76.

(11) Yamada, T. et al., Detection of wood discoloration in a canker fungus-inoculated Japan cedar by neutron radiography, J. Radioanalytical and Nuclear Chemistry, Vol. 264, No.2 (2005) pp.329-332.

(12) Matsubayashi, M., Study on development advanced neutron radiography imaging techniques, Doctor thesis of Kyoto Univ. (2004).

(13) Hardt, V. D. and Rottger, P. H., Neutron radiography handbook, D. Reidel Publ., Dordrecht, Holland (1981).

(14) Hibiki, T., Mishima, K. and Nishihara, H., Influence of scattered neutrons on void fraction measurement of two-phase flow using thermal neutron radiography, J. Nuclear Science and Technology, Vol. 34, No. 10 (1997), pp.996-1005.

(15) Yokoi, M. et al., Correction of scattering neutron effect in neutron CT image, Rept. KURRI-TR-359 (1992), pp.111-136 (in Japanese).

(16) Oda, M., Doctor thesis of Nagoya Univ. (1997) (in Japanese).

(17) Shepp, L. A. and Logan, B. F., The fourier reconstruction of a head section, IEEE Trans. Nuclear Science, Vol. 21, No.6 (1974), pp.21-43. 